\title{
The theory on thing's limits. Part 2: A brief analysis of the new knowledge of Newton's first law
}

\author{
Jian DING * \\ (Retired, Integrated Electronic Systems Lab Co. Ltd., Jinan 250100, China)
}

\begin{abstract}
According to the norm of identifying truth in this theory, and Newton's first law which is a basis that can look at the overall situation. By virtue of the electron storage ring as an experimental fact, it is pointed out: Only in reality can there be inertia, and vice versa. Inertia represents the continuity of the development of thing. As the speed gradually approaches to the $c$, the particle's mass also approaches to zero along with its static mass, which is the energy shrinkage effect of high-speed particles, and also the primary factor causing the spectrum redshift. Therefore, the Big Bang theory is wrong. All photons are produced from the high-density particles through electromagnetic radiations. Wherever there is fluctuation, there must be mass, and vice versa. This is the correct understanding of "wave-particle duality". No matter the high-speed electrons or the photons produced by them all have different static masses, but their charge-mass ratio is always the same physical constant, and not affected by relativistic effects and electromagnetic radiations. Which constitutes the internal mechanism of the uncertainty principle and conforms to the experimental facts related to it. It can be proved that in a constant magnetic field, the high-speed electron or photon with a relatively large curvature radius, which has a high moving speed and less mass, energy and wave frequency. Since Einstein used the absolute space-time established by Newton as the criterion and came to the conclusion that relative space-time was curved, then he should no longer make circular arguments, that was, used the relative space-time as the criterion, to change the unit length and time established by the absolute space-time.
\end{abstract}

Key words: mechanics; relativity; electron; photon; electromagnetic radiation; energy shrinkage PACS: 45.20.D-; 29.20.D-; 03.75.-b; 03.30.+p

\footnotetext{
* Corresponding author introduction: Jian DING, Male, Retired. Metaphysics and Physics. E-mail: jiandus@163.com
} 


\section{Introduction}

This article is the second part of the full text of "The theory on thing's limits". When quoting from the content of the full text's Part $1^{[1]}$, use "P1" to index. The rest may be inferred by analogy. In the P1, the characteristic of truth has been explained, that is, it cannot be proved by empirical methods, and can only be approached gradually by repeated practice. And according to the principle of inertia, the norm for identifying truth has been established. Based on this, we can break through the bondage of finite thinking, from the quantitative change of real space have gone deep into the qualitative change of ideal realm, and extend the philosophy of materialism to the category of metaphysics. Not only have restored the true nature of metaphysics, but also achieved the goal that Sir Isaac Newton pursued throughout his whole life. That was, theological (metaphysical) thought and scientific ideal were closely related, which was an organic whole, and the wisdom in one domain might enlighten the wisdom in another. ${ }^{[2]}$

This means that metaphysics has neither divorced from practices, nor just observed objective things with a one-sided, isolated and static way of thought. In people's daily life, everyone has used it, just not deliberately reflected on.

The contents contained in metaphysics, such as noumenon, axiom or postulation, absolute motion, etc., all can be called as the truths. They must have absoluteness and immutability, and do not exist in reality. So there is no direct connection among them, but they have the continuity with objective things in reality. That is to say, the differences between all truths and the objective things can reach an arbitrarily small value.

This means that once a certain truth is mastered, it is equivalent to standing in a position that can look at the overall situation. Thereby get rid of the dilemma of placing oneself in the midst of events, which is unable to look at the overall situation clearly, so difficult to grasp the correct direction of research, and always to use one-sided view to treat overall problem. At this time, again looking back at the objective things in reality, that is just like Sir Newton's famous saying: "If I have seen further than others, it is by standing upon the shoulders of giants."

As the introduction of the concept of absolute motion, Newton's first law of motion can be called as the starting point of classical physics, which has been widely applied to the physical space in reality, and is the best example. In other words, if compared with any one of the "giants" who only 
confined to the real physical space and determined to "gives no basis for the introduction of the concept of absolute motion" [3], he would be as always standing a little higher and seeing a little farther. Only in this way, it can be possible to realize the goal of mutual enlightenment between the natural science and metaphysics. ${ }^{[4]}$

\section{The truth contained in Newton's first law}

Newton's first law of motion, also known as the law of inertia. It is scientifically to clarify the two physical concepts of force and inertia, and correctly to explain the relationship between the force and the state of motion, as well as to put forward that all bodies have the property to keep its state of motion unchanged, that is inertia. It is expressed as ${ }^{[5]}$ : Everybody persevere in its state of rest, or of uniform motion in a right line, unless it is compelled to change that state by force impressed thereon.

Obviously, the body described therein is unaffected by forces, no matter it is stationary or moving along a straight line in uniform motion, its motion state is absolute (or ideal), does not exist in reality, and belongs to the category of metaphysics. And in reality, no matter what kind of physical problems you study, the law as a shadow always follows. It seems to prompt us to reflect on that the physical phenomena in reality are through what kind of a way does for the introduction of the concept of absolute motion provide a basis?

In this regard, the ideas and methods for the formation of "The theory on thing's limits", in P1, as well as the norm of identifying truth, in which discussions have already given the answer. That is to say, Newton's first law has also told us that only in real space can inertia exist, and vice versa. Inertia represents the continuity of the development of things. It can be inferred from this that as long as in reality, there must be causality between any two things. Next, use again the above-mentioned ideas, methods and norms to give a further discussion for several concepts involved in this law.

First of all, the "right line" defined in the law, which is an absolute straight line, does not exist in reality and belongs to the category of metaphysics. There are errors between any straight lines in reality and this absolute straight line. But there must be a straight line in reality, and the error between it and this absolute straight line can be an arbitrarily small value. Obviously, using this straight line in reality to standardize the space-time is the most accurate, but there are still errors after all. Therefore, the main use of this straight line is to abstract Newton's that absolute error-free straight line. 
So by the definition, the straight-line distance $\mathrm{S}$ is equal to the velocity $\mathrm{V}$ of uniform linear motion times the time spent $\mathrm{T}(\mathrm{S}=\mathrm{VT})$. The coordinate system thus established describes the absolute time and absolute space of Newtonian mechanics, which are referred to as absolute space-time for short. Only with this as the criterion, can we start from the consensus of absolute no error to understand the changes of objective things in reality.

Einstein since used the absolute space-time as the criterion and came to the conclusion that the relative space-time (i.e., the space-time in reality) was curved, he should no longer use the relative space-time as the criterion to change the unit length and unit time established by the absolute space-time. This kind of circular argument confused right and wrong, which was a logic fallacy, and even would deduce to turn back in time, so would not muddle through certainly in the face of truth. [3]

So why is the relative space-time curved? It is because of the existence of matter in there. With matter, there must be the interactions of forces among them. So according to Newton's first law, due to inertia, the linear motion of that has been bent or fluctuated, and the velocity also become uneven. From a macro perspective, you will feel that the space-time is curved, or the density uneven. And for this, various concepts such as mass, density, inertia, temperature, pressure, speed, frequency, wavelength, momentum, energy, etc., have been defined.

That is to say, as a truth, Newton's first law has told us that when the bidirectional reasoning is carried out between it and objective things, these concepts constitute our macro-cognitions of the different states in the reasoning processes. By virtue of the continuity of the objective things associated with them, there are causalities among these macro-cognitions, so that we can understand a certain objective thing more comprehensively, and would not produce the wrong cognitions that use one-sided view to treat overall problem, such as "Blind Men and the Elephant". ${ }^{[6]}$

\section{A particular case of Newton's first law}

This particular case has been mentioned in P1, which is the value $c$ of light speed in vacuum. In the absence of any external force, the photon always keeps moving along a straight line at a uniform velocity of $c=299792458(\mathrm{~m} / \mathrm{sec})$. This is the limit value of the speed in the universe, which is just higher an arbitrarily small value than the highest light speed in reality. This is an absolute motion and also an absolute frame of reference. A coordinate system which is admitted in mechanics is called as 
an "inertial system". Based on this, all inertial systems can be defined in reality, including the so-called stationary inertial systems. Just like the natural numbers and corresponding point positions on the number axis in P1, the abstractions of these inertial systems do not exist in reality, and belong to the absolute space-time view established by Newton.

Therefore, this particular case and therein the photon in uniform linear motion do not exist in reality. Such a photon is defined only to show that it has continuity with the photon which speed is closest to the $c$ in reality. From this it can be seen that Newton's first law is called as the law of inertia, which is precisely to emphasize that objects in reality have inertia. Where there is inertia, there must be matter and mass, and vice versa. But in view of the fact that truth must have absoluteness and immutability, so the noumena of various concepts belong to the metaphysical category, such as mentioned in this article, of inertia, mass, speed, fluctuation, momentum and energy, etc., among their noumena there are no continuity and any direct relationship. They can only be related through the objective things which have continuity with themselves in reality.

So, according to the norm for identifying truth in $\mathrm{P} 1$, what else is the difference between the photon that moves at the value $c$ of light speed in vacuum and a photon that moves at the highest light speed in reality?

As far as the former is concerned, which is the limit value that is starting from objective facts to be confirmed through the process of gradual approaching, does not exist in reality. Hence the former has neither mass $(m=0)$ nor energy $(E=0)$. And the latter is a photon in reality, of course, it has both mass $(m>0)$ and energy $(E>0)$. Otherwise, it means that there is no object of receiving force, so the characteristics of inertia certainly can not be shown.

Since in reality, the latter will inevitably be affected by external forces. According to Newton's first law, the linear motion has been become fluctuations, and the velocity also not uniform. This is precisely the result of the interaction between the external force and the inertial force of the latter having. As a result, the concept of "speed", which has been the velocity of moving along the direction of wavelength, also come into being with it. Obviously, this is exactly the objective reality we see. But more importantly, Newton's first law tells us that wherever there are fluctuations in reality, there must be matter and mass, and vice versa.

Furthermore, in view of the former that has no mass $(m=0)$, but has the characteristic of continuity with the latter, it can be realized that the mass of the latter is already very small. Here, 
Newton's first law seems to have given a instruction on how to grasp the correct direction of research. That is to say, as the speed of moving photon in reality gradually approaches to the $c$, its mass would gradually approach to zero, and the moving trajectory would gradually approach to the (absolutely) straight line, and the speed and velocity would approach to unity. This means that in reality, a photon with a relatively high moving speed should have less mass, energy, and wave frequency than another photon with a relatively low moving speed. Obviously, this is very consistent with the objective facts we have seen, such as the relationship between the energy and wave frequency of visible light.

In this regard, as early as the $1920 \mathrm{~s}$, the famous de Broglie matter wave relation ${ }^{[7]}$ has shown us that the momentum of a high-density particle (including electron or photon),

$$
\boldsymbol{p}=m \boldsymbol{v}=\frac{h}{\lambda}
$$

and its energy,

$$
E=m v^{2}=\frac{h v}{\lambda}=h f
$$

are related to its wavelength $\lambda$ or frequency $f$, but cannot grow without limit, and must be restricted by Planck's constant $h$. Among them, the moving speed along the direction of wavelength $v=\lambda f$. As the cornerstone of the expansion of Newtonian mechanics to the wave mechanics or quantum mechanics, the matter wave relation can be through the continuity with objective things in reality, to rely on each other with Newton's three laws of motion and the value $c$ of light speed in vacuum, and produce causality.

\section{Photons are the products of electromagnetic radiation}

It is indisputable that the photon in reality has mass $(m>0)$. If there is no mass $(m=0)$, it means that there is no matter. That is to say, there is no object of receiving force, of course, there will be no inertia. As a result, those physical phenomena that should be related to it in classical mechanics, such as inertia, fluctuation, temperature, pressure, momentum and energy, etc., will not appear. Because the interaction among matters is a necessary condition for us to perceive the above-mentioned physical phenomena. On the contrary, as long as you find anything related to these physical phenomena, it must be related to mass.

As far as photon is concerned, it is high-speed particle that has been produced by electromagnetic radiation from a high-density particle, such as electron, proton, neutron, or atomic 
nucleus, etc. Einstein defined it as the quantum of light, and later it has been called as the photon for short. According to the kinetic energy formula of his special relativity ${ }^{[8]}$,

$$
E_{k}=E-E_{0}=m_{0} c^{2}(\gamma-1)
$$

the word "quantum" contains indivisibility between matter and energy, while the word "light" is focused on electromagnetic radiation. That is to say, all high-speed particles that are produced from the high-density particles through electromagnetic radiation, should be belonged to the category of photons.

The formula (3) also contains the mass-energy formula,

$$
E=m c^{2} \text { or } \Delta E=\Delta m c^{2}
$$

and mass-speed formula,

$$
m=m_{0} \gamma
$$

of his special relativity. Among them, $E_{0}=m_{0} c^{2}$ is the static energy and $E$ the total energy, both are aimed at the same moving object or particle. And the mass-speed formula (5) reveals that the concept of mass is divided into two parts along with the moving speed $v$ of objects (including various high-density particles) changes. In the above formulas, $m$ is the mass to represent inertia, $m_{0}$ is the static mass to represent the quantity of matter, $\gamma=1 / \sqrt{1-v^{2} / c^{2}}$ is the expansion factor, and $c$ is the value of light speed in vacuum.

As far as photons are concerned, since you can perceive their fluctuations and energy, according to the previous analysis of Newton's first law, and the formulas (1) and (2), should know that they have inertia and mass. This is the correct understanding of "wave-particle duality". Of course, nor would be for the sake of whether light exactly is a wave or a particle to be a state of endless debate and each airs his own views. As for photons that have no mass but energy, such a paradox that confuses the two domains of definition, no one would be to believe it anymore.

In fact, the problem is not so simple. Take random electromagnetic radiation as an example. According to the formulas (4) and (5), the energy that every one of radiated photons has will take away a part of the static mass (that is, a certain amount of matter). In this regard, there has been no consensus. And this is exactly the objective fact that modern physics must face, any so-called authoritative statements that have attempted to circumvent it vaguely, all will certainly come back 
here again, and reconfirm. Otherwise, modern physics will be fettered here and difficult to make great progress.

In 1927, German physicist Werner Karl Heisenberg first pointed out that the more precisely the position of some particle was determined, the less precisely its momentum could be known, and vice versa. The principle can also be expressed as: When the momentum $\boldsymbol{p}$ is determined, the speed $\boldsymbol{v}$ (or position) cannot be determined; when the speed $\boldsymbol{v}$ (or position) is determined, the momentum $\boldsymbol{p}$ cannot be determined.

In Heisenberg's time, physicists generally believed that an electron would not be further broken down. For a low-speed moving in non-relativistic state, the charge, (static ) mass, and charge-mass ratio of each electron all were considered to be physical constants, which was a consensus that people formed one hundred years ago. And now we must realize that due to the effect of electromagnetic radiation, the charge and static mass of each electron both have been no longer the constants.

For the electrons with different static masses, their charge-mass ratio $e / m_{0}$ is always the same physical constant. When they are moving at different speeds, the momentums or energies can be the same. So the phenomenon presented is "When the momentum $\boldsymbol{p}$ is determined, the speed $\boldsymbol{v}$ cannot be determined." Conversely, when they are moving at the same speed, the momentums or energies would be various. So the phenomenon presented is "When the speed $v$ is determined, the momentum $\boldsymbol{p}$ cannot be determined." This is precisely the internal mechanism of the uncertainty principle. ${ }^{[9]}$

\section{The energy shrinkage effect of high-speed particles}

Now we must realize that if continue to insist the static mass of every electron as a constant, the continuities among them will be lost. Only by abandoning this wrong view can we ensure that the uncertainty principle will not offend Newton's first law. That is to say, regarding whether an electron will be broken down further, it should be verified carefully.

In reality, along with the moving speed $v$ of a high-speed particle (electron or photon) gradually approaches to the $c$, the same particle exhibits two effects: on the one hand, according to the formula (5) its mass $m$ keeps getting bigger; and on the other hand, its static mass $m_{0}$ keeps getting smaller due to the loss caused by electromagnetic radiation. The result of final synthesis shows that its mass gradually approaches to zero along with its static mass. This is the energy shrinkage effect of 
high-speed particles. ${ }^{[10]}$

For example, the electron storage rings widely in use at present, are the large-scale scientific facilities for studying high-energy physics ${ }^{[11]}$. In the storage ring, an electron momentum ${ }^{[12]}$,

$$
\boldsymbol{p}=m \boldsymbol{v}=e \boldsymbol{B} R
$$

can be obtained directly from the equation of the centripetal force $m v^{2} / R$ and Lorentz force $e \boldsymbol{B} \boldsymbol{v}$. In formula 6 , e is the charge of an electron, $\boldsymbol{B}$ is the magnetic induction intensity, and $R$ is the curvature radius of the moving electron in the ring.

For high-speed electrons, the relativistic effects should be considered. By formula 5 and 6 , the relative speed

$$
\beta=\frac{v}{c}=\sqrt{\frac{\left(\frac{e}{m_{0}}\right)^{2}}{\left(\frac{e}{m_{0}}\right)^{2}+\left(\frac{c}{\boldsymbol{B} R}\right)^{2}}}
$$

of an electron can be deduced. In view of the fact that the magnetic induction intensity $\boldsymbol{B}$ and curvature radius $R$ in the storage ring have been designed to the calibration values, and the charge-mass ratio of electrons is a constant, it can be calculated by formula (7) that the speed $v$ of every moving electron therein is also a calibration value, and has nothing to do with its mass.

As a result, these high-speed electrons that perhaps have different static masses, can move along the circular orbit to do cyclotron motion at a given speed $v$ in the tubular vacuum chamber of the storage ring. Under normal circumstances, the lifetimes of the electron beam current in the storage ring can generally last for several hours. Once the several hours to have passed, the electron beam current will quickly decay to zero. The reason is that in these several hours, most of the static mass $\sum \Delta m_{0}$ belonged originally to each electron itself has been gradually lost as electromagnetic radiation turned into photons. At this time, the static mass $m_{0}$ of each electron in the storage ring all has become very small. As a result, when a photon radiated, its impulse has been transferred to the electron and enough pushes the electron to the inner wall of the tubular vacuum chamber of the storage ring. ${ }^{[13]}$

In the above example, by virtue of the widely used electron storage ring as an experimental fact, has proved that due to electromagnetic radiation, the charge e of a high-speed electron would follow along with its static mass $m_{0}$ to be lost synchronously, and its charge-mass ratio $e / m_{0}$ remains 
unchanged. This is the energy shrinkage effect of high-speed electrons. The nature of this matter is serious and of great significance, in order to being cautious, which should be verified to facilitate consensus. The behavior of any attempt to circumvent or muddle through, may all deduce the wrong conclusion, or cause the lost of research direction. In fact, it is not difficult to verify. As long as you do interference experiments on the electron beam currents in the storage ring before and after the several hours separately, and no need to quantify, just compare the wavelengths of the two qualitatively to distinguish them.

The experimental facts have also revealed that the electron mass $M_{0}$ and elementary charge $e_{0}$ in the fundamental physical constants only refer in particular to the statistical values of low-speed electrons that just reach the status where they can leave the atoms. The ratio of the two is the charge-mass ratio $e_{0} / M_{0}$ of the fundamental physical constants. Due to the existence of energy shrinkage effect of every high-speed electron, in general, its static mass and charge are in the state of synchronous reduction, but the charge-mass ratio,

$$
e / m_{0}=e_{0} / M_{0}
$$

has remained unchanged. Therefore, it must be emphasized that the charge-mass ratio $e / m_{0}$ of an electron refers to the ratio of its charge to the quantity of matter, which is not affected by relativistic effects and electromagnetic radiation. By the same token, this conclusion also applies to every photon that has been radiated by the electrons in the storage ring.

\section{A minimal error or deviation may result in wide divergence}

Then, these photons must follow the law of motion determined by formula (7). That is to say, under the premise of the given magnetic induction intensity $\boldsymbol{B}$, the photon with a relatively large curvature radius $R$ must have a relatively high moving speed and relatively less mass, energy and wave frequency. This is consistent with the experiment of decomposing sunlight with a triangular prism, and indicating that the visible light is also composed of the charged particles of different sizes. In other words, compared with the purple light, the photons of red light have relatively high moving speeds, which should be an objective fact that Newton's first law has tacitly approved.

Therefore, it is emphasized again that the energy shrinkage effect of high-density particles caused by electromagnetic radiation is an objective reality that must be faced in modern physics. Combined with Formula (1) and (2), it can be seen that this is exactly the primary factor causing the 
spectrum redshift. However, in view of the fact that most mainstream thinkings have been still restricted to the cognition that an elementary particle (electron or photon) will not be broken down further, and disturbed by the uncertainty principle, so which has not caused enough attention. This primary factor causing the spectrum redshift has actually been replaced by the Doppler Effect, and transformed into that celestial bodies are moving away from us. A minimal deviation may result in wide divergence. But now, the cosmological redshift seems to have been judged as a correct theory while think carefully, a little worry involuntarily... ${ }^{[14]}$

That is to say, just like the moral in the story "Blind Men and the Elephant", the well-known Big Bang theory is a cognitive error that has been used by one-sided view to treat overall problem. The reason for such a error, if traced to the source, still because it has offended the most fundamental truth in Physics, that is, Newton's first law. What on earth has been offended? It is inertia, that is, the continuity of the development of things. Only in real space can inertia exist, and vice versa. That is to say, there must be causality between any two things in reality. Otherwise, it would be beyond the scope of real space. It should be noted here that only mathematics has the possibility to run through two different domains of definition, that is, both continuity and discontinuity can exist in that.

Take the uncertainty principle as an example. This is a research conclusion that has been drawn from a large number of experimental facts and reached a consensus. If continue to insist the static mass of every electron as a constant, so the price paid is that the continuity among them will be lost, that is, offending Newton's first law. Therefore, such a paradox that celestial bodies were moving away from us, would be deduced. This means that the conclusion made can only be that the static mass of every electron being different. Yes, it not only conforms to a large number of experimental facts, but also gets the acquiescence of Newton's first law, and everything becomes harmonious.

It was because they did not stand on this basis that could look at the overall situation, so these physicists would have ignored the objective fact around themselves, that was, electromagnetic radiation could cause an electron to be broken down further. From the two conceptual ambiguity for inertia and charge-to-mass ratio, to the uncertainty principle by muddling through, and even the photon that had no mass but with energy, and so on, various similar "authoritative theories" have wandered in modern physics. And once confronting with the judgment of truth, the behavior of any attempt to circumvent or muddle through, will inevitably become the victim of one's own mistake. Although this process may be long, it is insignificant compared to eternal truth. 
Incidentally, as a light source of electromagnetic radiation, the speed of moving electrons in the storage ring, $v>0.99 c$, which is sufficient to prove that the value $c$ of light speed in vacuum is independent of the state of motion of the emitting body.

\section{Conclusion}

This article is according to the ideas and methods for the formation of "The theory on thing's limits", in P1, as well as the norm of identifying truth, its characteristic is based on Newton's first law, which is equivalent to standing in a position that can look at the overall situation. Thereby get rid of the dilemma of placing oneself in the midst of events, which is difficult to grasp the correct direction of research and always to use one-sided view to treat overall problem. The theory is applicable to all academic categories, and can be combined with objective practices. In terms of testing authoritative theories, clarifying chaos, and deriving new knowledge, etc., there are the following conclusions:

1. Newton's first law is called as the law of inertia, which is precisely to emphasize that objects in reality have inertia. Where there is inertia, there must be matter and mass, and vice versa. Inertia, which represents the continuity of the development of things. Only in reality can there be inertia, and vice versa. That is to say, there must be causality between any two things in reality. Otherwise, it would be beyond the scope of real space, that is, offending Newton's first law.

2. Now that Einstein used the absolute space-time established by Newton as the criterion and came to the conclusion that the relative space-time (i.e., the space-time in reality) was curved, he should no longer use the relative space-time as the criterion to change the unit length and unit time established by the absolute space-time. This kind of circular argument confused right and wrong, which was a logic fallacy, so would not muddle through certainly.

3. There are matters in the space-time in reality, so there must be the interactions of forces among them. According to Newton's first law, due to inertia, the linear motion of matter has been become fluctuations. Therefore, wherever there are fluctuations, there must be matter and mass, and vice versa. As far as photons are concerned, now that we perceive their fluctuations and energy, should know that they have inertia and mass. This is the 
correct understanding of "wave-particle duality".

4. The value $c$ of light speed in vacuum is a particular case of Newton's first law. Based on this, it can be deduced that in reality, a photon with a relatively high moving speed should have less mass, energy, and wave frequency than another photon with a relatively low moving speed. This is consistent with the objective facts we have seen. Because every one of photons that has been radiated by the electron in the storage ring, has the same charge-mass ratio as electron, but their static masses are different from one another. Then, these photons must follow the law of motion determined by formula (7). That is to say, under the premise of the given magnetic induction intensity $\boldsymbol{B}$, the photon with a relatively large curvature radius $R$ must have a relatively high moving speed. This is consistent with the experiment of decomposing sunlight with a triangular prism, that is, compared with the purple light, the photons of red light have a relatively high moving speed.

5. The photons in reality, which are high-speed particles produced by high-density particles through electromagnetic radiation. According to the formulas (4) and (5), the energy that the radiated photons have will take away a part of the static mass. Therefore, by virtue of the widely used electron storage ring as an experimental fact, it has been proved that due to electromagnetic radiation, the charge e of a high-speed electron would follow along with its static mass $m_{0}$ to be lost synchronously, and its charge-mass ratio $e / m_{0}$ remains unchanged. This means that the charge and static mass of electron both have been no longer the constants.

6. For the electrons with different static masses, when they are moving at different speeds, the momentums or energies can be the same. So the phenomenon presented is "When the momentum $\boldsymbol{p}$ is determined, the speed $\boldsymbol{v}$ cannot be determined." Conversely, when they are moving at the same speed $\mathrm{v}$, the momentums or energies would be various. So the phenomenon presented is "When the speed $\boldsymbol{v}$ is determined, the momentum $\boldsymbol{p}$ cannot be determined." This is precisely the internal mechanism of the uncertainty principle, which not only ensures the continuity among things in reality, also has been supported by a large number of experimental data that when the principle was approved.

7. In reality, along with the moving speed $v$ of a high-speed particle (electron or photon) 
gradually approaches to the $c$, the same particle exhibits two effects: on the one hand, according to the formula (5) its mass $m$ keeps getting bigger ; on the other hand, its static mass $m_{0}$ keeps getting smaller due to the loss caused by electromagnetic radiation. The result of final synthesis shows that its mass would follow along with its static mass gradually to approach to zero. This is the energy shrinkage effect of high-speed particles, and also the primary factor causing the spectrum redshift. Therefore, the Big Bang theory is wrong. The nature of this matter is serious and of great significance, which is an objective reality that must be faced in modern physics, and should be verified to facilitate consensus.

8. As a light source of electromagnetic radiation, the speed of moving electrons in the storage ring, $v>0.99 c$, which is sufficient to prove that the value $c$ of light speed in vacuum is independent of the state of motion of the emitting body.

\section{References}

[1] Jian DING. 2021. "The Theory on Thing's Limits. Part 1: The Norm of Identifying Truth.” OSF Preprints. May

\section{1. doi:10.31219/osf.io/gh4vk}

[2] Wen H W. Study on the Newtonian religious ideas [D]. Jinhua in China: Zhejiang Normal University, 2009: 16. DOI: 10.7666/d.y1549479 (in Chinese)

[3] Albert Einstein. Relativity [A]. Fang Z Q, Han W B, He W G. Out of My Late Years by Albert Einstein [M]. Hainan in China: Hainan Press; 2000: 41, 57. (in Chinese)

[4] Jian DING. The Research of Using Truth to Restrict Authoritative Theories. Journal of Philosophy and Ethics. 2020; 2(1): 43-50.

[5] Isaac Newton. The Mathematical Principles of Natural Philosophy [M]. Wang K D. Xian: Shaanxi Peoples Publishing House, 2001: 18. (in Chinese)

[6] Sakyamuni. Dirghagama-sutra (Vol. 19) [M]. Buddhayasas, Zhu Fonian. Beijing: Sino-Culture Press, 2013: 609-610. (in Chinese)

[7] Louis de Broglie. Physics and Microphysics [M]. Zhu J D. Beijing: The Commercial Press, 1992: 54. (in Chinese) 
[8] Albert Einstein. On the Electrodynamics of Moving Bodies [A]. Xu L Y, Fan D N. The collected works of Einstein (Vol. 2) [C]. Beijing: The Commercial Press, 1977: 83-118. (in Chinese)

[9] Ding J. A brief analysis of the research scheme of the cyclotron radiation from a single electron. 2020; 4(2):

60-64. DOI: 10.15406/paij.2020.04.00202

[10] Jian D, Xiuqin HU. Piercing the veil of modern physics: part $1 \&$ basics. Physics \& Astronomy International Journal. 2018; 2(2): 128-134. DOI: 10.15406/paij.2018.02.00074

[11] Jin Y M. Electron Storage Ring Physics [M]. Hefei: USTC Press, 2001: 1. (in Chinese)

[12] Wu S C, Wang Z Q. Modern Physics Experiment [M]. Beijing: Peking University Press, 1995: 151. (in Chinese)

[13] Ding Jian, Hu Xiuqin. The Electro-Ultimate Particles and a New Method for Detecting the Photon Static Mass. International Journal of Modern Physics and Application, 2014, 1(1): 1-8.

[14] S W Hawking. A Brief History of Time [M]. Changsha: Hunan Science \& Technology Press, 1996: 46-67. (in Chinese) 


\title{
物极理论 2: 牛顿第一定律新知浅析 \\ 丁健*
}

\author{
积成电子股份有限公司（已退休） 中国济南 250100
}

\begin{abstract}
䐱夏: 依据该理论中认定真理的规范. 立足于牛顿第一定律这个总摸全局的基点。借助电子储存环作为实 验事实, 指出: 只有在现实中, 才会有惯性, 反之亦然。惯性, 代表了事物发展的连续性。随若速率逐渐 地向 $c$ 趋近. 粒子的质量也随若其静质量不断地向 0 趋近, 这就是高速粒子的能量收缩效应. 也是引起光谱 红移的首要因素。因此. 大㩧炸理论是错误的。所有光子都是由高密度粒子通过电磁辐射产生的。凡是有 波动的垉方. 必定有质量. 反之亦然。这才是对 “波粒二象性” 的正确认知。无论是高速电子, 还是由其 产生的光子. 它们的静质量都各不相同, 但荷质比却为同一个物理常数. 且不受相对论效应和电磁辐射的 影响。这构成了不确定性原理的内在机制, 并符合与之相关的实验事实。可以证明, 在佰定磁场中. 曲率 半径相对较大的高速电子或光子, 具有较高的运动速率和较小的质量、能量和波动频率。爱因斯坦既然用 牛顿所确立的绝对时空为淮绳, 得出相对时空是弯曲的结论, 就不应该再循环论证. 即以相对时空为淮绳. 去改变绝对时空所确立的单位长度和时间。
\end{abstract}

关键词: 力学; 怢义相对论; 电子; 光子; 电磁辐射; 能量收缩

中图分类号: 0301;0572.21+1;0413.1;0536;0412.1

\section{The theory on thing's limits. Part 2: A brief analysis of the new knowledge of Newton's first law Jian DING \\ (Retired, Integrated Electronic Systems Lab Co. Ltd Jinan 250100, China)}

\begin{abstract}
According to the norm of identifying truth in this theory, and Newton's first law which is a basis that can look at the overall situation. By virtue of the electron storage ring as an experimental fact, it is pointed out: Only in reality can there be inertia, and vice versa. Inertia represents the continuity of the development of thing. As the speed gradually approaches to the $c$, the particle's mass also approaches to zero along with its static mass, which is the energy shrinkage effect of high-speed particles, and also the primary factor causing the spectrum redshift. Therefore, the Big Bang theory is wrong. All photons are produced from the high-density particles through electromagnetic radiations. Wherever there is fluctuation, there must be mass, and vice versa. This is the correct understanding of "wave-particle duality". No matter the high-speed electrons or the photons produced by them all have different static masses, but their charge-mass ratio is always the same physical constant, and not affected by relativistic effects and electromagnetic radiations. Which constitutes the internal mechanism of the uncertainty principle and conforms to the experimental facts related to it. It can be proved that in a constant magnetic field, the high-speed electron or photon with a relatively large curvature radius, which has a high moving speed and less mass, energy and wave frequency. Since Einstein used the absolute space-time established by Newton as the criterion and came to the conclusion that relative space-time was curved, then he should no longer make circular arguments, that was, used the relative space-time as the criterion, to change the unit length and time established by the absolute space-time.
\end{abstract}

Key words: mechanics; relativity; electron; photon; electromagnetic radiation; energy shrinkage PACS: 45.20.D-; 29.20.D-; 03.75.-b; 03.30.tp

- 作者简介：丁健 (1953-)，男，已退休. 主要研究方向: Metaphysics and physics... E-mail: iiandus@163.com 